\title{
Joint Data Detection and Phase Recovery for Downlink MC-2D-CDMA Systems
}

\author{
Rui Fa, Member, IEEE, and Pei Xiao, Member, IEEE
}

\begin{abstract}
The performance of a downlink synchronous MC-CDMA system with joint frequency-time domain spreading (MC-2D-CDMA) is investigated in this paper. We propose a two dimensional adaptive minimum mean square error (MMSE) receiver, which works in decision-directed mode after an initial training period. A subcarrier phase tracker, which comprises a bank of phase locked-loops (PLLs), is employed in the receiver to track the fading phase variability. Furthermore, a simplified phase tracker structure is proposed to reduce the system complexity. The performance of the data detector and the behavior of the phase tracker are analyzed theorectically in this paper and are shown to match the simulation results. Both analysis and simulation indicate that the proposed system outperforms the conventional MC-DS-CDMA systems by exploiting frequency diversity and facilitating subcarrier synchronization.
\end{abstract}

Keywords - MC-2D-CDMA, adaptive receiver, two dimensional spreading, phase locked loops, frequency-selective Rayleigh fading

\section{INTRODUCTION}

$\mathbf{M}$ ULTICARRIER CDMA (MC-CDMA) technique has become increasingly popular in wireless communications, mainly due to its high spectral efficiency, robustness to frequency selective fading and flexibility to support integrated applications. Various MC-CDMA schemes have been proposed and can be classified into two main categories based on two different ways of combining CDMA and Orthogonal Frequency Division Multiplexing (OFDM) [1], [2]. One is to spread the original data sequence in the time domain; and the other is to spread in frequency domain. The former scheme, which is so called MC-DS-CDMA [3], has some advantages, such as easy tracking of the fading process over subcarriers. It, however, cannot achieve the frequency diversity gain, which is the main advantage of using multicarrier modulation technique. The latter scheme, which is usually called MCCDMA, can exploit frequency diversity by employing combining strategies such as Maximum Ratio Combining (MRC), Orthogonality Restoring Combining (ORC), Equal Gain Combining (EGC) and MMSE Combining since the fading of each subcarrier is different. However, accurate knowledge of the channel state information (CSI) is critical for these combining strategies to function efficiently. The performance of MC-CDMA in frequency selective fading channels has been analyzed in several papers, e.g., in [4], and reference therein. However, some of them assume perfect subcarrier

Dr. Rui $\mathrm{Fa}$ is with the department of Electronics, the University of York, York Y010 5DD, UK and Dr. Pei Xiao is with the Institute of Electronics, Communications and Information Technology (ECIT), Queens University Belfast, BT3 9DT, UK. E-mails: rf533@ohm.york.ac.uk, pei.xiao@qub.ac.uk phase recovery at the receiver, while others employ channel estimation schemes that require information of all active users, including their spreading codes and power levels, which is not available in the downlink at the mobile terminals [5]. Without this information, the receiver performs poorly, or even fails to function in multi-user scenario since the chipbased interference from other users on each subcarrier makes the subcarrier phase tracking impossible. Phase recovery is required by any communication system employing coherent reception. Various types of PLLs, which are widely used in phase and frequency synchronization tasks [7], have been analyzed in [6] - [9]. The performance of first-order PLL has also been investigated in the presence of phase noise, residual multiple access interference (MAI), and additive white Gaussian noise (AWGN) for DS-CDMA based systems [6]. The nonlinear Fokker-Planck method is usually employed to derive the probability density function (PDF) of the phase estimation error [9]. However, to our knowledge, PLL performance analysis in fading channels with Doppler frequency shifts has not been discussed in the existing literature. In this paper, we provide a theoretical performance analysis for the second-order PLL over a time-varying Rayleigh fading channel using the Fokker-Planck method, and derive the phase estimate error PDF and variance.

Recently, Zheng, et.al, proposed an MC-CDMA system employing two dimensional, joint frequency and time domain spreading [10], known as MC-2D-CDMA. It was shown in [10] that by combining MC-CDMA and MC-DS-CDMA, and thus exploiting both time and frequency diversities, the new system performs better than the conventional MC-CDMA system, especially in fast fading channels. In this paper, we show that this MC-2D-CDMA scheme not only achieves both time and frequency diversity gains, but also greatly facilitates subcarrier synchronization, which is crucial for coherent reception. We propose a two dimensional adaptive MMSE multiuser receiver using the Normalized Least Mean Square algorithm (NLMS) for the considered MC-2D-CDMA system. To comply with the downlink transmission requirements, the proposed receiver performs data detection and subcarrier recovery without other users' information (including spreading codes).

Iterative receiver structures utilizing turbo processing principle [11] have attracted a great deal of attention in recent years for DS-CDMA and MC-CDMA systems [12] - [14]. These techniques greatly improve the receiver performance by exchanging extrinsic information between the detector and channel decoder. However, most of the existing iterative multiuser detection and decoding schemes are only applicable for uplink systems, since all users' signature waveforms and 
other information are required [12]. The performance of third generation systems may well be limited by the performance of the downlink. Therefore, it is important to develop effective iterative algorithms to improve the system performance in the downlink transmission. In this paper, we propose a joint iterative detection and phase recovery scheme, in which the proposed receiver feeds the a priori information from softinput soft-output (SISO) decoder back to update both the MMSE filter coefficients and phase locked-loops (PLLs). We also investigate how the subcarrier phase tracker which comprises a bank of second-order PLLs benefits from the iterative process over the time-varying frequency selective fading channel.

The rest of the paper is organized as follows. In Section II and Section III, the MC-2D-CDMA system is briefly described and the proposed 2D MMSE receiver structure as well as the iterative detection and phase recovery schemes are presented. The performance analysis of the MMSE detection and the PLL phase tracking for the studied MC-2D-CDMA system is conducted in Section IV. Analytical and simulation results are given in Section V. Finally, conclusions are drawn in Section VI.

\section{SYSTEM DESCRIPTION}

\section{A. MC-2D-CDMA Transmitter Model}

Fig. 1 shows the structure of the tranmitter for a $K$ user downlink synchronous MC-2D-CDMA system employing Quadrature Phase Shift Keying (QPSK) modulation. The data sequence $\left\{b_{k}(i)\right\}$ from the $k$ th user are encoded into coded bits $\left\{u_{k}(i)\right\}$, which are subsequently interleaved and each block of two coded and interleaved bits $\left\{v_{k}^{0}(i), v_{k}^{1}(i)\right\}$ is mapped into one of the four QPSK symbols $d_{k}(i)$ using Gray mapping scheme. The interleaver and deinterleaver are denoted as $\Pi$ and $\Pi^{-1}$, in Fig. 1 and Fig. 4, respectively. Preambles with length $P$ that are known to the receiver are inserted in front of every $G$ data symbols. They serve as pilot symbols for both MMSE detector and the subcarrier phase tracker on the receiver side. Each QPSK symbol is multiplied by a frequency spreading code $\mathbf{c}_{k}^{f}$ (the subscript $k$ is the user index, and the subscript $f$ or $t$ is used to differentiate between frequency and time domain) with spreading factor $M$. The resulting sequence after spreading is $\mathrm{S} / \mathrm{P}$ converted in parallel over $M$ subcarriers. Each subcarrier signal is multiplied by a time spreading code $\mathbf{c}_{k}^{t}$ with spreading gain $N$, and $c_{k}^{f}[m], c_{k}^{t}[n] \in\{-1,+1\}, m=$ $1, \ldots, M, n=1, \ldots, N$. After joint frequency-time spreading, the signal is modulated by an OFDM modulator, which is implemented using the inverse fast Fourier transform (IFFT) operation. A cyclic prefix is inserted with duration longer than the impulse response of the channel to combat intersymbol interference (ISI) and intercarrier interference (ICI). In this paper, we consider both uncoded and coded systems. In the uncoded case, the encoding and interleaving steps are left out, i.e., the information sequence $\left\{b_{k}(i)\right\}$ is directly mapped to QPSK symbols. The rest of the spreading and modulation process remain the same as in the coded system described previously. The complex envelope of the transmitted signal for $k$ th user due to the transmission of the symbol $d_{k}(i)$ can

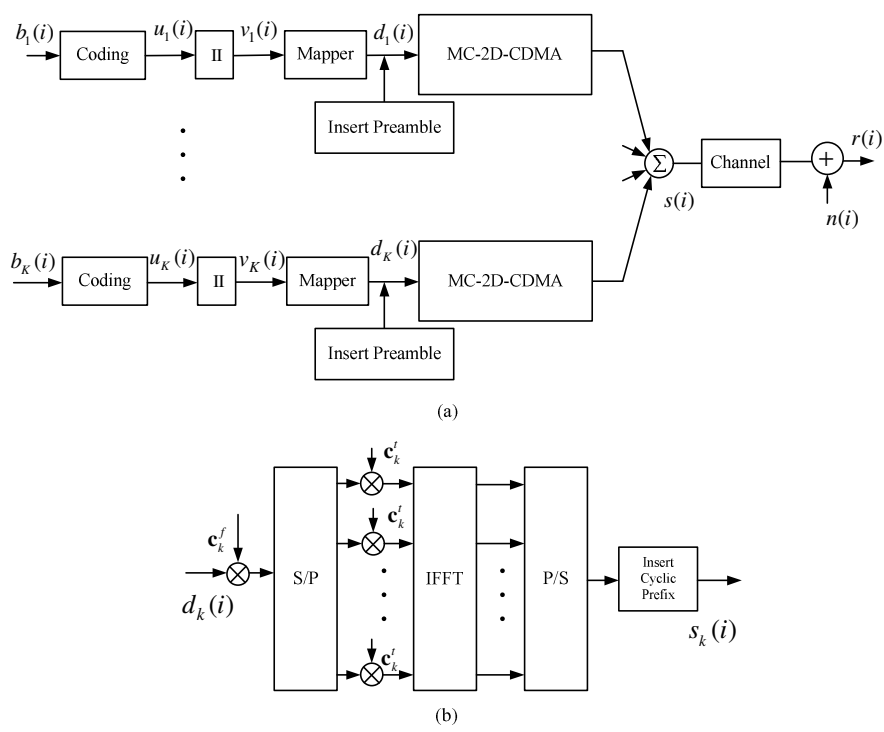

Fig. 1. (a) The diagram of the transmitter for the coded MC-2D-CDMA system with $K$ users, (b) MC-2D-CDMA structure.

be expressed as

$$
s_{k}(t)=\sum_{m=1}^{M}\left[c_{k}^{f}(m) \sum_{n=1}^{N} d_{k}(i) c_{k}^{t}(n) g\left(t-n T_{c}\right)\right] e^{j 2 \pi f_{m} t},
$$

where $g(t)$ is a rectangle pulse during $\left[0, T_{c}\right]\left(T_{c}\right.$ denotes the chip duration), and $f_{m}$ is the $m$ th subcarrier frequency.

\section{B. Channel Model}

We consider a downlink $L$-tap multipath channel with complex equivalent low-pass time-variant impulse response

$$
h(t, \tau)=\sum_{l=1}^{L} \alpha_{l}(t) \delta\left(\tau-\tau_{l}\right),
$$

where $\alpha_{l}(t)$ is the complex channel gain for the $l$ th path. It is a complex Gaussian random process; $\tau_{l}$ is propagation delay for the $l$ th path, and $\delta(\cdot)$ is the Dirac delta function. If the channel delay spread is shorter than the duration of the cyclic prefix, each subcarrier experiences flat fading. The channel gain for the $m$ th subcarrier, which is denoted as $H_{m}$, can be expressed as [10]

$$
H_{m}(t)=\sum_{l=1}^{L} \alpha_{l}(t) e^{-j 2 \pi f_{m} \tau_{l}}=\rho_{m}(t) e^{j \theta_{m}(t)},
$$

where the $\rho_{m}$ and $\theta_{m}(t)$ are the equivalent amplitude and phase shift, respectively, for the $m$ th subcarrier. The former is assumed to be highly correlated Rayleigh random variables for different subcarriers; the latter is assumed to be uniformly distributed over the interval $[0,2 \pi)$, and also correlated in time and frequency. The received signal at the mobile terminal can thus be expressed as

$$
\begin{aligned}
r(t) & =\sum_{k=1}^{K}\left\{\sum_{m=1}^{M} \rho_{m}(t) c_{k}^{f}(m)\left[\sum_{n=1}^{N} d_{k}(i) c_{k}^{t}(n) g\left(t-n T_{c}\right)\right]\right. \\
& \left.\times e^{\left(j 2 \pi f_{m} t+\theta_{m}(t)\right)}\right\}+n(t),
\end{aligned}
$$




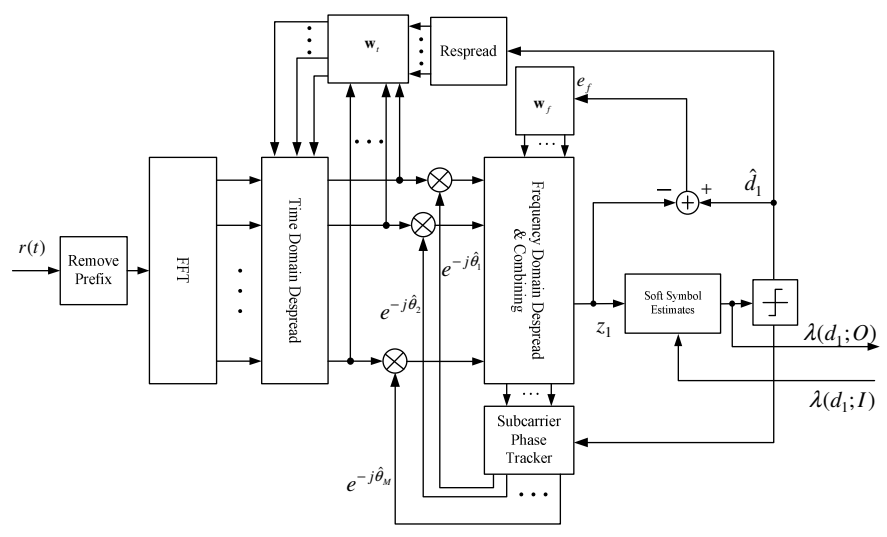

Fig. 2. Proposed receiver structure for MC-2D-CDMA.

where $n(t)$ is the additive white Gaussian noise with zero mean and variance $N_{0}$.

\section{PROPOSED RECEIVER MODEL}

\section{A. Two-Dimensional MMSE Receiver}

A block diagram of the proposed two-dimensional MMSE receiver is shown in Fig 2. Without loss of generality, the first user $(k=1)$ is assumed to be the user of interest. The received signal is first fed into the OFDM demodulator to remove the cyclic prefix and to be processed by FFT. We assume that the fading process is slow enough so that the channel amplitude and phase remain constant duration the transmission of one symbol. The post-FFT received vector for the $m$-th subcarrier can be written as

$$
\boldsymbol{r}_{m}(i)=\rho_{m}(i) e^{j \theta_{m}(i)} \sum_{k=1}^{K} c_{k}^{f}(m) d_{k}(i) \boldsymbol{c}_{k}^{t}+\boldsymbol{v}_{m}(i),
$$

where $\boldsymbol{v}_{m}(i)$ is AWGN vector at the $m$ th subcarrier.

Each of the $M$ received vectors is processed by a time domain MMSE filter $\boldsymbol{w}_{k, m}^{t}$ to form $\hat{z}_{k, m}=\boldsymbol{w}_{k, m}^{t}(i)^{H} \boldsymbol{r}_{m}(i)$, for $m=1,2, \ldots, M$. The filter $\boldsymbol{w}_{k, m}^{t}(i)$ is designed to minimize the mean-squared error

$$
\begin{aligned}
\mathcal{J}_{m} & =E\left(\left|e_{1, m}^{t}(i)\right|^{2}\right) \\
& =E\left(\left|e^{j \theta_{m}(i)} c_{1}^{f}(m) d_{1}(i)-\boldsymbol{w}_{1, m}^{t}(i)^{H} \boldsymbol{r}_{m}(i)\right|^{2}\right),
\end{aligned}
$$

and the NLMS solution can be derived as [15]

$$
\boldsymbol{w}_{1, m}^{t}(i+1)=\boldsymbol{w}_{1, m}^{t}(i)+\frac{\tilde{\mu}_{t}}{a_{t}+\left\|\boldsymbol{r}_{m}(i)\right\|^{2}} \boldsymbol{r}_{m}(i) e_{1, m}^{t}(i)^{*},
$$

where $(\cdot)^{*}$ denotes the conjugate operation, $\tilde{\mu}_{t}$ is the step size and $a_{t}$ is a small non-negative constant.

The vector $\hat{z}_{1}=\left[\hat{z}_{1,1}, \ldots, \hat{z}_{1, M}\right]^{T}$ is subsequently processed by a phase corrector and a frequency domain MMSE filter $\boldsymbol{w}_{1}^{f}(i)$, yielding

$$
\hat{b}_{1}(i)=\operatorname{sgn}\left(\boldsymbol{w}_{1}^{f}(i)^{H} \boldsymbol{z}_{1}(i) e^{-j \hat{\boldsymbol{\theta}}(i)}\right),
$$

where $\hat{\boldsymbol{\theta}}=\left[\begin{array}{lll}\hat{\theta}_{1} & \ldots & \hat{\theta}_{M}\end{array}\right]$ denotes the estimated subcarrier phase vector. The frequency domain filter $\boldsymbol{w}_{1}^{f}$ minimizes the mean-squared error

$$
\mathcal{J}=E\left(\left|e_{1}^{f}(i)\right|^{2}\right)=E\left(\left|d_{1}(i)-\boldsymbol{w}_{1}^{f}(i)^{H} \boldsymbol{z}_{1}(i) e^{-j \hat{\boldsymbol{\theta}}(i)}\right|^{2}\right),
$$

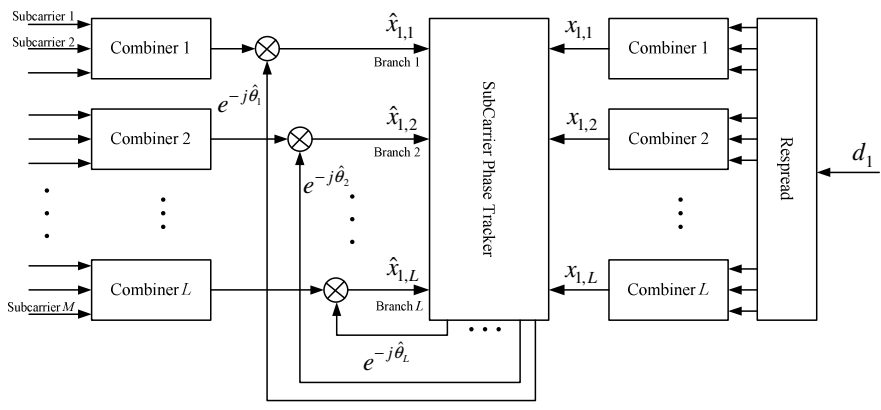

Fig. 3. Structure of simplified subcarrier phase tracker.

leading to the solution [15]

$$
\boldsymbol{w}_{1}^{f}(i+1)=\boldsymbol{w}_{1}^{f}(i)+\frac{\tilde{\mu}_{f}}{a_{f}+\left\|\boldsymbol{z}_{1}(i)\right\|^{2}} \boldsymbol{z}_{1}(i) e^{-j \hat{\boldsymbol{\theta}}(i)} e_{1}^{f}(i)^{*},
$$

where $\tilde{\mu}_{f}$ is the step size for the frequency domain filter and $a_{f}$ is a small non-negative constant.

The subcarrier phase tracker comprises a bank of phase locked loops (PLLs), which can be expressed mathematically as

$\hat{\theta}_{m}(i+1)=\hat{\theta}_{m}(i)+K_{1} \varphi_{m}(i)+K_{1} K_{2} \sum_{n=0}^{i-1} \varphi_{m}(n), m=1, \ldots, M$

where $\varphi_{m}(i)=\operatorname{Im}\left\{z_{k, m}(i) \hat{z}_{k, m}(i)^{*}\right\}$ is the phase-error measurement, and $K_{1}, K_{2}$ are PLL control parameters, which are always set to be small constant values, and $z_{k, m}(i)$ is defined to be $z_{k, m}(i)=c_{k}^{f}(m) d_{k}(i)$ in training mode or $z_{k, m}(i)=c_{k}^{f}(m) \hat{b}_{k}(i)$ in decision-directed mode.

\section{B. Simplified Phase Tracker Scheme}

Due to the correlation among the subcarriers, the phase of several neighbouring fading branches can be tracked by only one PLL. Therefore, the number of PLLs is reduced, and so is the complexity of the system. The proposed simplified phase tracker scheme is shown in Fig. 3. Suppose $M$ is the multiple of the number of groups $L$, the index $m$ in (11) becomes $l=1, \ldots, L$ and $\varphi_{l}(i)=$ $\operatorname{Im}\left\{x_{k, l}(i) \hat{x}_{k, l}(i)^{*}\right\}$, and $x_{k, l}=\boldsymbol{c}_{k, l}^{f}{ }^{T} \boldsymbol{c}_{k, l}^{f} d_{k}(i)$ in training mode or $x_{k, l}=\boldsymbol{c}_{k, l}^{f}{ }^{T} \boldsymbol{c}_{k, l}^{f} \hat{b}_{k}(i)$ in decision-directed mode. $\hat{x}_{k, l}(i)=\boldsymbol{w}_{k, l}^{f}(i)^{H} \hat{z}_{k, l}(i)$, and $\boldsymbol{c}_{k, l}^{f}, \boldsymbol{w}_{k, l}^{f}(i), \hat{\boldsymbol{z}}_{k, l}(i)$ denote the lth sub-vectors of $\boldsymbol{c}_{k}^{f}, \boldsymbol{w}_{k}^{f}(i), \hat{\boldsymbol{z}}_{k}(i)$, respectively, corresponding to the $l$ th branch of grouped subcarriers. The optimum performance is obtained if the number of PLLs is equal to number of subcarriers. However, its complexity becomes higher compared to the simplified scheme. Obviously, there is a trade-off between complexity and performance.

\section{Iterative detection for the coded system}

The iterative structure for the coded system is shown in Fig. 4. It consists of two stages: an adaptive 2D MMSE detection, followed by a SISO channel decoding. The two stages are linked by a de-mapper, de-interleaver, interleaver and mapper. As illustrated in Fig. 4, the sequence of the soft-symbol 


\section{PERFORMANCE ANALYSIS}

\section{A. BER performance analysis}

In this section, the BER expression of MC-2D-CDMA is derived based on the Gaussian approximation and MMSE criterion. For the simplicity of analysis, the $0^{\text {th }}$ symbol from the first user is supposed to be detected. We can re-write the received $2 \mathrm{D}$ spread signal in a vector/matrix format as

$$
r=C A d+n,
$$

where $\boldsymbol{n}$ denotes complex Gaussian noise vector with zero mean and covariance matrix $\boldsymbol{\Gamma}=\frac{N_{0}}{2 T} \boldsymbol{I} \in \mathbb{R}^{N M \times N M}$ ( $T$ is the symbol duration, $\mathbb{R}$ denotes the real field), and

$$
\begin{aligned}
\boldsymbol{C}= & {\left[\boldsymbol{c}_{1}, \boldsymbol{c}_{2}, \ldots, \boldsymbol{c}_{K}\right], } \\
\boldsymbol{c}_{k}^{\prime f}= & {\left[c_{k}^{f}(1) H_{1}, \ldots, c_{k}^{f}(M) H_{M}\right]^{T}, } \\
\boldsymbol{c}_{k}^{\prime \prime}= & {\left[c_{k}^{t}(1), \ldots, c_{k}^{t}(M)\right]^{T}, } \\
\boldsymbol{c}_{k}= & {\left[\mathbf{c}_{k}^{\prime f}(1) \mathbf{c}_{k}^{t}(1), \ldots, \mathbf{c}_{k}^{\prime f}(1) \mathbf{c}_{k}^{t}(N),\right.} \\
& \left.\ldots, \mathbf{c}_{k}^{\prime f}(M) \mathbf{c}_{k}^{t}(1), \ldots, \mathbf{c}_{k}^{\prime f}(M) \mathbf{c}_{k}^{t}(N)\right]^{N M \times 1}, \\
\boldsymbol{A}= & \operatorname{diag}\left(A_{1}, \ldots, A_{k}\right), \quad \boldsymbol{d}=\left[d_{1}(0), \ldots, d_{K}(0)\right]^{T} .
\end{aligned}
$$

The optimum filter is derived using the well-known WienerHopf solution $\boldsymbol{w}_{\text {opt }}=\boldsymbol{R}^{-1} \boldsymbol{p}$, where $\boldsymbol{R}=\mathrm{E}\left[\mathbf{r r}^{H}\right]$ represents the correlation matrix of the vector $\boldsymbol{r}$, and $\boldsymbol{p}=\mathrm{E}\left[\mathbf{r} d_{1}^{*}(0)\right]$ denotes the cross-correlation vector. The optimum weight vector maximizes the output signal to interference plus noise ratio (SINR) of the receiver [16]

$$
\begin{aligned}
\mathbf{S I N R}_{\text {max }} & =\frac{\left\|\boldsymbol{w}_{o p t}^{H} \boldsymbol{c}_{k}\right\|^{2}}{\boldsymbol{w}_{o p t}^{H} \Gamma \boldsymbol{w}_{o p t}+\left\|\boldsymbol{w}_{o p t}^{H} \overline{\boldsymbol{C}}_{k} \overline{\boldsymbol{A}}_{k}\right\|^{2}} \\
& =A_{1}^{2} \boldsymbol{c}_{k}^{H}\left(\overline{\boldsymbol{C}}_{k} \overline{\boldsymbol{A}}_{k} \overline{\boldsymbol{C}}_{k}^{H}+\Gamma\right)^{-1} \boldsymbol{c}_{k},
\end{aligned}
$$

where $\bar{C}_{k}$ and $\bar{A}_{k}$ denote the matrix $\bar{C}$ and $\bar{A}$ without the $k$ th column. Since the MAI plus noise at the output of the filter is approximately Gaussian as shown in [17], the probability of error is given by

$$
P_{e}(H)=Q\left(\sqrt{2 \mathbf{S I N R}_{\max }}\right)
$$

The average BER over all channel realizations is thus obtained by

$$
P_{a v}^{k}=\int_{H} P_{e}(H) f(H) d H
$$

where $f(H)$ represents the joint probability density function of the channel coefficients. Since the closed form expression of (24) is not tractable, we obtain $P_{a v}^{k}$ semi-analytically by averaging $P_{e}(H)$ calculated by (23) using a long sequence of fading channel coefficients. Finally, the BER performance is obtained by averaging $P_{a v}^{k}$ over all active users, i.e.,

$$
\mathbf{B E R}=\frac{1}{K} \sum_{k=1}^{K} P_{a v}^{k} .
$$

\section{B. PLL performance analysis}

In this section, a statistical analysis of the effect of the fading, residual MAI and phase error on the decision-directed PLL for the proposed iterative receiver is given. The analog representation of second order PLL in (11) can be written as [9]

$$
\varphi(t)=A \sin \phi(t)+n^{\prime}(t)
$$

where $A$ is a constant, $\phi(t)$ is the phase error and $n^{\prime}(t)$ is the equivalent noise, which combines residual MAI and phase noise. Here we assume that $n^{\prime}(t)$ exhibits a Gaussian distribution. According to (11), we may obtain the loop filter transfer function $F(s)=1+K_{2} / s$ and the phase-locked-loop differential equation as

$$
\begin{aligned}
\frac{d \phi(t)}{d t}=\frac{d \theta(t)}{d t} & -K_{1}\left[A \sin \phi(t)+n^{\prime}(t)\right] \\
& -K_{1} K_{2} \int_{0}^{t}\left[A \sin \phi(u)+n^{\prime}(u)\right] d u
\end{aligned}
$$

where $\theta(t)$ is channel phase process. At low signal-to-noise ratios (SNRs), a more realistic approach to derive the steadystate PDF of the phase estimator is to use the Fokker-Planck method. In the previous analysis of the phase estimator error based on the Fokker-Planck method, $d \theta(t) / d t$, the rate of phase variation was considered to be a constant [9] or was assumed to be an additive noise with zero mean [6]. Compared with the previous derivation in [6], [9], we treat $d \theta(t) / d t$ as a stochastic process corresponding to the Rayleigh fading channel and introduce $m(t)$ assuming that

$$
\frac{d \theta(t)}{d t}=K_{2} m(t)+\frac{d m(t)}{d t} .
$$

Thus, equation (27) can be decomposed into two equations as

$$
\begin{gathered}
\frac{d \epsilon(t)}{d(t)}+A K_{1} \int_{0}^{t} \sin \phi(u) d u=m(t)-K_{1} \int_{0}^{t} n^{\prime}(u) d u \\
\frac{d^{2} \epsilon(t)}{d t^{2}}+A K_{1} \sin \phi(t)=\frac{d m(t)}{d t}-K_{1} n^{\prime}(t) .
\end{gathered}
$$

where $\epsilon(t)$ is introduced to solve the equation and it is assumed to be $\phi(t)=K_{2} \epsilon(t)+\frac{d \epsilon(t)}{d t}$ [9]. Using the same methodology as presented in [9] to solve the two-dimentional Fokker-Plank equation, we may obtain the stationary density function as

$$
\frac{d}{d \phi}\left(\left\{\Phi+\Omega-K_{2} M\right\} p(\phi)+\frac{1}{2} \sum_{k=0}^{1} \sum_{l=0}^{1} X_{k l} \frac{d p(\phi)}{d \phi}\right)=0
$$

where

$$
\begin{aligned}
& \Phi=A K_{1} \sin \phi \\
& \Omega=A K_{1} K_{2} \int E[\sin \phi(t+\tau) \mid \phi(t)] d \tau, \\
& M=E[m(t) \mid \phi(t)], \\
& \quad=E[\theta(t)]-E\left[K_{2} e^{-K_{2} t} \int_{0}^{t} \theta(u) e^{K_{2} u} d u\right]=0, \\
& \sum_{k=0}^{1} \sum_{l=0}^{1} X_{k l}=\lambda_{g}+\lambda_{d},
\end{aligned}
$$




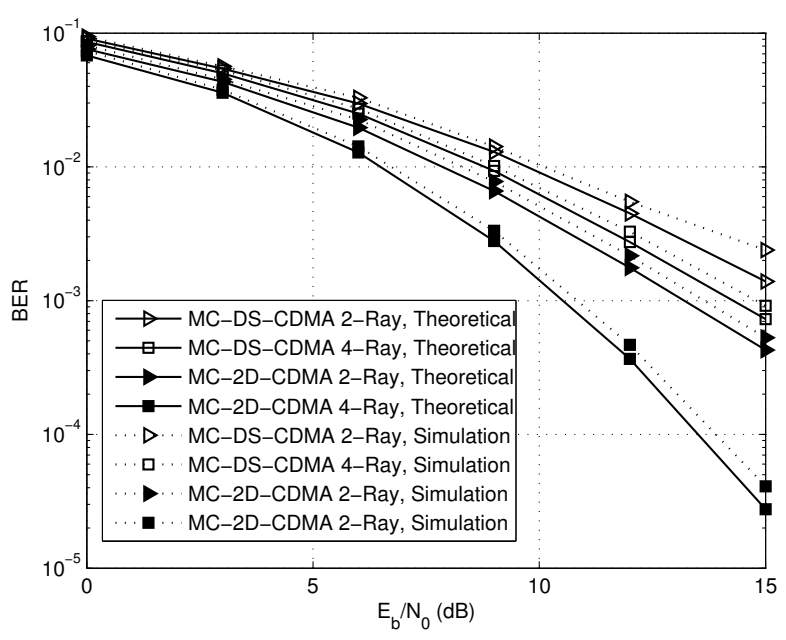

Fig. 5. Theoretical performance vs. simulation results with perfect phase tracking of MC-2D-CDMA and MC-DS-CDMA, the vehicle speed is $60 \mathrm{~km} / \mathrm{h}$.

where

$$
\lambda_{g}=\lim _{\Delta t \rightarrow 0} \frac{K_{1}^{2}}{\Delta t} \int_{t}^{t+\Delta t} \int_{t}^{t+\Delta t} n^{\prime}(u) n^{\prime}(v) d u d v=\frac{K_{1}^{2} N_{0}}{2}
$$

and

$$
\lambda_{d}=\lim _{\Delta t \rightarrow 0} \frac{\overline{[\Delta \theta(t)]^{2}}}{\Delta t}=-\left.2 \frac{d R_{\theta}(\tau)}{d \tau}\right|_{\tau \rightarrow 0^{+}}
$$

where where $R_{\theta}(\tau)$ is the autocorrelation function of channel phase process defined as in (32) where $f_{d} T$ is the normalized doppler frequency and $J_{0}(\cdot)$ is the $0^{\text {th }}$ order Bessel function of the first-kind [18] . Following a series of substitutions, we may obtain (31e) as

$$
\sum_{k=0}^{1} \sum_{l=0}^{1} X_{k l}=6 \sqrt{2} f_{d} T+\frac{K_{1}^{2} N_{0}}{2}
$$

Thus, (30) is approximated to [9]

$$
\frac{d}{d \phi}\left\{\frac{2 A K_{1}}{\lambda_{d}+\lambda_{g}}\left[\sin \phi\left(\frac{A K_{1}}{A K_{1}+K_{2}}\right)\right] p(\phi)+\frac{d p(\phi)}{d \phi}\right\}=0 .
$$

Solving (34) yields the Tikhonov distribution, the PDF of the phase estimator error becomes

$$
p(\phi) \approx \frac{\exp (\beta \cos \phi)}{2 \pi J_{0}(\beta)} .
$$

where $\beta$ denotes the equivalent loop SNR expressed as

$$
\beta=\frac{A^{2}}{\left(12 \sqrt{2} f_{d} T / K_{1}^{2}+N_{0}\right) B_{L}},
$$

where $B_{L}$ denotes the loop-noise bandwidth. Furthermore, at a large equivalent loop SNR, the PDF of the phase estimator error can be approximated by a Gaussian distribution and the variance of the phase error becomes

$$
\sigma_{\phi}^{2} \approx \frac{1}{\beta}=\frac{\left(12 \sqrt{2} f_{d} T / K_{1}^{2}+N_{0}\right) B_{L}}{A^{2}} .
$$

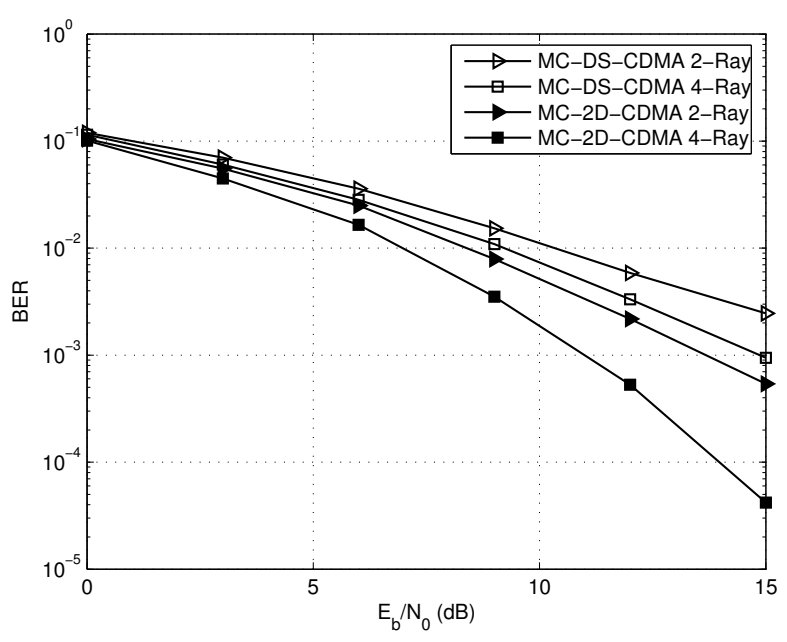

Fig. 6. BER vs. SNR performance comparison between MC-2D-CDMA and MC-DS-CDMA with phase tracking.

\section{SIMULATION RESULTS}

In this section, both analytical and simulation results are presented to demonstrate the effectiveness of the proposed schemes. The number of users is assumed to be 15; the walsh code of length 16 is used for both time domain and frequency domain spreading; the number of subcarriers is 16 . The fading process is generated by driving a zero-mean complex Gaussian random process through a designed filter, the power spectrum density (PSD) of which is shaped as classic Doppler PSD. Four channels are considered: the first two have simple 2-ray and 4-ray multipath delay profiles with propagation delays equal to $10 T_{c}$ and $5 T_{c}$, respectively; and the other two types of channel are modelled as correlated Rayleigh channels with correlation coefficients 0.5 and 0.8 , respectively ${ }^{1}$, and generated using the method in [19]. For PLLs, the parameters are chosen to be $K_{1}=0.7, K_{2}=0.1 * K_{1}$ in training mode; and $K_{1}=0.5$, $K_{2}=0.1 * K_{1}$ in decision directed mode, respectively. The preamble length is set to be 100 symbols, which are followed by 500 data symbols. The chip rate employed is $3.84 \mathrm{Mchips} / \mathrm{s}$, as specified in the 3GPP standard. Unless otherwise stated, we consider a normalized Doppler frequency of $f_{d} T=0.001$, where $T$ is the duration of a symbol. The system operates at a carrier frequency of $f_{c}=2 \mathrm{GHz}$ and vehicle speeds are simulated with a maximum Doppler frequency of $f_{d, \max }=120$ $\mathrm{Hz}$, which corresponds to a nominal velocity of $v=60 \mathrm{~km} / \mathrm{h}$. The step sizes in the 2D MMSE filter are set to $\tilde{\mu}_{t}=0.1$ and $\tilde{\mu}_{f}=0.9$ since they in general work better than other values for the studied system.

It has already been shown in [10] that MC-2D-CDMA outperforms MC-CDMA. The emphasis of this paper is the comparison between MC-2D-CDMA and MC-DS-CDMA. Fig. 5 shows the BER versus SNR performance comparison between theoretical analysis and simulation results with perfect phase tracking of the proposed MC-2D-CDMA system. The former is obtained by evaluating (23), (24) and (25). It is compared with that of the conventional MC-DS-CDMA system, for

\footnotetext{
${ }^{1}$ The correlation is imposed on each pair of contiguous subcarriers.
} 


$$
R_{\theta}(\tau)=\frac{3}{2 \pi} \sin ^{-1}\left[J_{0}\left(2 \pi f_{d} T \tau\right)\right]+6\left\{\frac{1}{2 \pi} \sin ^{-1}\left[J_{0}\left(2 \pi f_{d} T \tau\right)\right]\right\}^{2}-\frac{3}{4 \pi^{2}} \sum_{n=1}^{\infty} \frac{\left[J_{0}\left(2 \pi f_{d} T \tau\right)\right]^{2 n}}{n^{2}}
$$

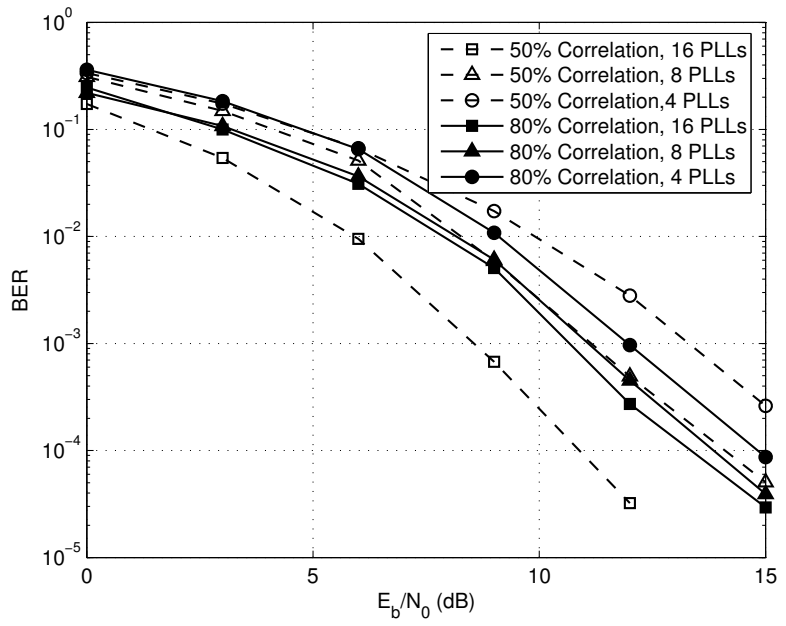

Fig. 7. Performance of MC-2D-CDMA with different numbers of PLLs in correlated Rayleigh fading channels.

which the length of spreading codes and the number of subcarriers are both set to be 16, for fair comparison. The theoretical analysis conducted in Section IV-A also applies to MC-DS-CDMA systems except that the spreading vectors in (21c), (21d) are defined differently. Each subcarrier data stream of MC-2D-CDMA is based on the chip-spread signal; whereas for MC-DS-CDMA, it is based on symbol-spread signal. Thus, the frequency diversity can be more readily exploited by the 2D structure and consequently, the BER performance improves. Comparing to MC-DS-CDMA, MC2D-CDMA achieves a gain of $3 \mathrm{~dB}$ at the target $\mathrm{BER}=10^{-3}$ for both 2-Ray and 4-Ray channels. Furthermore, the simulation results with perfect phase tracking match the theoretical performance rather well.

Fig. 6 presents the BER performance comparsion between MC-2D-CDMA and MC-DS-CDMA based on the simulation results without the assumption of perfect phase tracking. More than $3 \mathrm{~dB}$ gain is observed in 4-ray fading channel and $2 \mathrm{~dB}$ gain in 2-ray fading channel, respectively. For both channels, due to the phase noise introduced by the phase tracker at the low SNR level, the simulation results are worse than the theoretical ones with the assumption of perfect phase tracking shown in Fig. 5.

In Fig. 7, we investigate the performance of proposed receiver with different numbers of PLLs in a correlated Rayleigh fading channel. One can see that when the correlation factor is low (50\%), the proposed receiver with a full number of PLLs (the number of PLLs is equal to the number of subcarriers, which is 16) performs well, while when the number of PLLs decreases, the performance degrades rapidly. On the other hand, in the case of high $(80 \%)$ correlation, the performance of proposed receiver degrades only by $1 \mathrm{~dB}$, when comparing

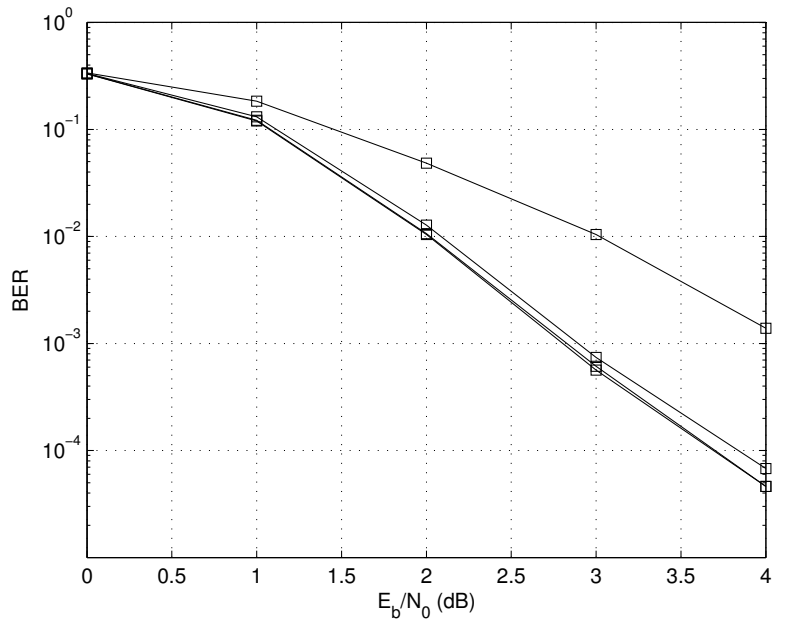

Fig. 8. Performance of the iterative detection and phase recovery for the coded MC-2D-CDMA system. The topmost curve represents the 1st stage detection and decoding, the bottom curve represents the 4th stage detection and decoding.

4 PLLs to 16 PLLs. Furthermore, only one PLL is enough in the flat fading case (100\% correlation). In practical systems, the multicarrier systems experience highly correlated fading, and the proposed simplified subcarrier phase tracker can be employed for complexity reduction.

Fig. 8 shows the performance of iterative detection and phase recovery for the coded MC-2D-CDMA system. All the users employ a rate $1 / 2$ convolutional code with constraint length 5 and generator polynomials $(23,35)$ in octal form. Each user employs a different random interleaver with a block size equal to length of 6000 encoded bits. The first iteration can be viewed as a system without feedback and in the subsequent iterations, the 2D MMSE filter coefficients and the phase tracker are updated using the feedback from the channel decoder. It is observed that the iterative algorithm converges after 3 iterations, beyond which the performance improvement is negligible. This indicates that the latency introduced by the iterative process is moderate. Clearly, significant improvement can be achieved by applying iterative process if we compare the topmost curve representing the performance of one time detection and decoding with the bottommost curve representing the performance of the proposed iterative scheme upon convergence. We also observe that most significant gains are obtained at the second iteration, and the gain at each iteration increases with increased SNR. In particular, a $1.5 \mathrm{~dB}$ of gain is observed at the third iteration at target BER level of $10^{-3}$ compared to the initial iteration without feedback.

In Fig. 9 and Fig. 10, we investigate the PDF of the phase estimation error for MC-2D-CDMA over the fading channel with normalized Doppler frequency $f_{d} T$ equal to 0.001 . The SNR is fixed to be $E_{b} / N_{0}=5 \mathrm{~dB}$. It is obvious to see from 


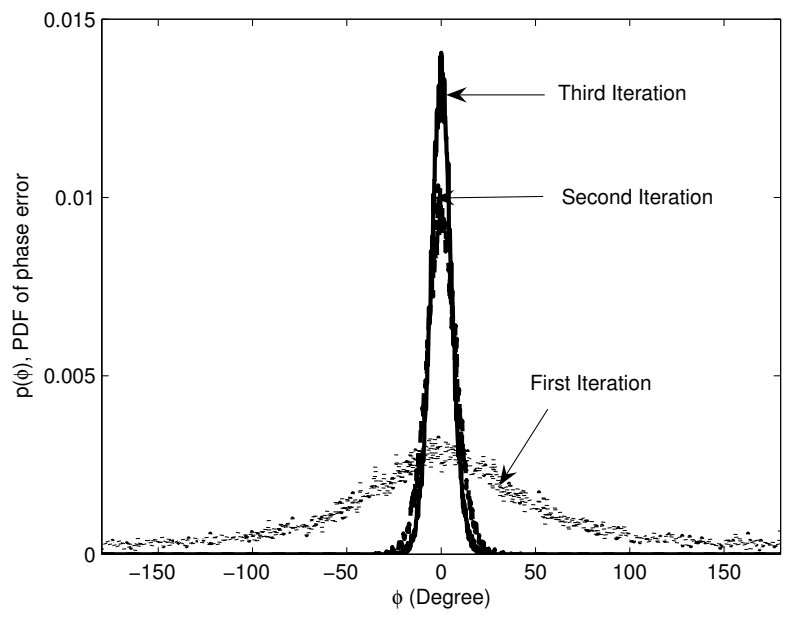

Fig. 9. PDF of the phase estimation error for the iterative scheme.

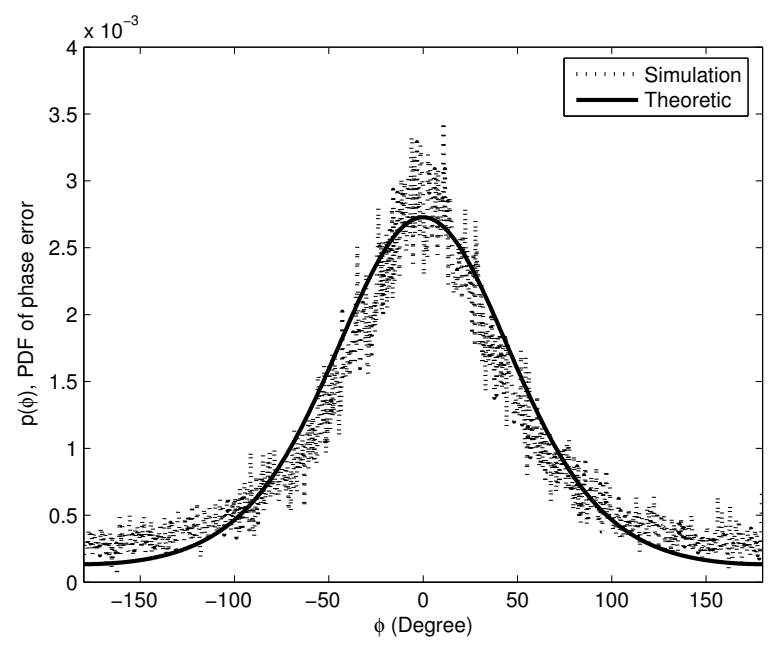

Fig. 10. PDF of the phase estimation error at the first iteration: simulation vs. analysis.

Fig. 9 that the iterative scheme significantly suppresses the combined residual MAI and phase noise, the variance of which decreases with increasing number of iterations. The figure also indicates that the phase estimation error can be approximated as a Gaussian random variable, which concurs with the results shown in [9]. Fig. 10 shows that the simulation results for the PDF of phase error fluctuate around the analytical values, which means the PLL performance analysis conducted in Section IV-B is fairly accurate, it provides an effective measure to predict the PLL performance for the MC-2D-CDMA system under investigation.

\section{CONCLUSION}

MC-CDMA systems with two-dimensional spreading in the presence of time-variant frequency-selective fading and unknown MAI are investigated in this paper. We proposed an adaptive two dimensional MMSE receiver with subcarrier phase tracker, which comprises a bank of PLLs. The BER performance of the MC-2D-CDMA system and phase estimation error of the PLLs are analyzed theoretically. The analyses are validated by the Monte-Carlo simulations and proved to be fairly accurate. Both simulation and analytical results indicate that the MC-2D-CDMA system obtains better performance than the conventional MC-DS-CDMA. We also show that the proposed simplified phase tracker performs better in fading conditions with higher correlation coefficients than those with lower correlation coefficients; and in coded MC-2D-CDMA systems, the iterative process significantly improves the performance of the adaptive detector, and enhances the tracking capability of PLLs.

\section{REFERENCES}

[1] Hara, S. and R. Prasad, "Overview of multicarrier CDMA," IEEE Commun. Mag., 1997. 35(12): p. 126-133.

[2] Linnartz, J., "Performance analysis of synchronous MC-CDMA in mobile Rayleigh channel with both delay and Doppler spreads," IEEE Trans. Veh. Tech., 2001. 50(6): p. 1375-1387.

[3] Liu, H. and H.J. Yin, "Receiver design in multicarrier direct-sequence CDMA communications," IEEE Trans. Commun., 2001. 49(8): p. 14791487.

[4] Hara, S. and R. Prasad. "Design and performance of multicarrier CDMA system in frequency-selective Rayleigh fading channels," IEEE Trans. Veh. Tech., 1999. 48(5): p. 1584-1595.

[5] Kalofonos, D.N., M. Stojanovic, and J.G. Proakis, "Performance of adaptive MC-CDMA detectors in rapidly fading Rayleigh channels," IEEE Trans. Wireless Commun., 2003. 2(2): p. 229-239.

[6] Huang, W., I. Andonovic, and M. Nakagawa, "PLL performance of DSCDMA systems in the presence of phase noise, multiuser interference, and additive Gaussian noise," IEEE Trans. Commun.,1998. 46(11): p. 1507-1515.

[7] J. Proakis, Digital Communication, Third Edition. New York: McGrawHill, 1998.

[8] Charles F. J. and W. C. Lindsey, "Some analytical and experimental phase-locked loop results for low signal-to-noise ratios," IEEE Proceedings.,1966. 54(9): p. 1152-1166.

[9] A.J. Viterbi, Principles of Coherent Communication, 1966, New York: McGraw-Hill.

[10] Zheng, K., G.Y. Zeng, and W.B. Wang, "Performance analysis for OFDM-CDMA with joint frequency-time spreading," IEEE Trans. Broad., 2005. 51(1): p. 144-148.

[11] J. Hagenauer. "The Turbo principle: tutorial introduction and state of the art". Proc. International Symposium on Turbo Codes, pp. 1-11, Sept. 1997.

[12] Wang, X.D. and H.V. Poor, "Iterative (Turbo) soft interference cancellation and decoding for coded CDMA." IEEE Trans. Commun., May, 1997, pp. 1634-1638.

[13] P. Xiao, E. Ström, "Correction of extrinsic information for iterative decoding in a serially concatenated multiuser DS-CDMA system". IEEE Trans. on Wireless Commun., vol. 5, no. 3, pp. 591-602, March 2006.

[14] V. Poor, "Iterative multiuser detection". IEEE Signal Process. Magazine, vol. 21, pp. 81-88, Jan 2004.

[15] S. Haykin. Adaptive Filter Theory, 4th edition, Prentice Hall, 2002.

[16] Madhow, U. and M.L. Honig, "MMSE Interference Suppression for Direct-Sequence Spread- Spectrum," IEEE Trans. Commun., 1994. 42(12): p. 3178-3188.

[17] V. Poor, S. Verdu. "Probability of error in MMSE multiuser detection", IEEE Trans. Inform. Theory, vol. 43, no. 3, pp. 858-871, May 1997.

[18] W.C. Jakes, Microwave Mobile Communications, IEEE Press, New York, 1974,.

[19] Natarajan B., C.R. Nassar and V. Chandrasekhar, "Generation of correlated Rayleigh fading envelopes for spread spectrum applications," IEEE Commun. Lett., 2000. 4(1): p. 9-11. 